\title{
Virtual Simulations for Drone Education of Senior High School Students
}

\author{
Julia Dominic P. Espinola, Jose Emmanuel D. Ignacio, John Peter L. Lacaden, Charles Brian D. \\ Toribio and Alvin Y. Chua
}

\begin{abstract}
Drone technology is further increasing in popularity and demand and drone education is already implemented in certain college courses. This paper presents a virtual simulation module that will cater to the needs of the senior high school level and that would lead to a module that is essentially less technical and more interactive. The virtual simulations module will be an integrated website which contains fundamental information on what a drone is and how to operate one. It will also incorporate an interactive game simulation wherein the drone model created from $3 D$ printing will be integrated in. To verify the achievement of the goals of this paper, the researchers will conduct a survey among a randomized pool of senior high school students, which will include a pre and post-test about the module. Through this surveys that the participants answered, the researchers used t-test methodology to conclude that the module is indeed effective on delivering the information to the students with up to 90\% positive response about the module. Additionally, the researchers have taken into account the preferences and suggestions of the students who participated.
\end{abstract}

Index Terms: drones, educational module, website, virtual simulation

\section{INTRODUCTION}

Drones were primarily created for military purposes. Though earlier references to military drones in popular culture do exist, the period from 2010 to the present has seen the drone make more appearances in popular culture at a far more frenetic pace than ever before [1]. Drones nowadays, especially quadcopters, are used in various fields of media, especially in photography, over more practical applications such as search-and-rescue operations, structural surveillance, wildlife preservation and monitoring against poachers, etc. [2]. Because of this rise in the popularity of quadcopters, it is important that the population using this technology is aware of its components, basics and operation.

In the Philippines, the regulations regarding UAV follow the Civil Aviation Authority of the Philippines (CAAP), under the Department of Transportation. Since this

Revised Manuscript Received on September 22, 2019.

Julia Dominic P. Espinola, Integrated School, De La Salle University, Manila, Philippines, julia_espinola@dlsu.edu

Jose Emmanuel D. Ignacio, Integrated School, De La Salle University, Manila, Philippines, phose_emmanuel_ignacio@dlsu.edu

John Peter L. Lacaden, Integrated School, De La Salle University, Manila, Philippines, phjohn_peter_lacaden@dlsu.edu.ph

Charles Brian D. Toribio, Integrated School, De La Salle University, Manila,Philippines, charles_toribio@dlsu.edu.ph

Alvin Y. Chua, Mechanical Engineering Department, De La Salle University, Manila, Philippines, alvin.chua@dlsu.edu.ph technology is still relatively new to the country, the regulations are still subject to change as time progresses [3]. Although rules and safety precautions are already in place, drone education in the country remains limited. The study of drones is taught mostly in college and universities, on a technical level usually aligned with engineering courses. But because of the $\mathrm{K}$ to 12 Curriculum implemented in 2016 by the Department of Education, courses taken in first and second year college are brought down to grades 11 and 12 in Senior High School so as to provide the students ample time for mastery of different subjects [4]. Different learning techniques have been developed for Senior High School, particularly the online learning platforms. Studies have shown that on average, students perform better when participating in digital classes instead of traditional ones [5], while others show that students learn more by "doing", as in John Dewey's theory on Experiential Learning [6].

The popularity of drones in the recreational field has been growing since the early 2010s. From being primarily used in the military, drones are now being used in photography and racing, professional and not [7]. Smaller "toy" drones have been made available for civilian use. Should the module created by the researchers be proven successful, more people will be able to understand the process and concept behind drones and their basic functions and components [8], [9].

For De La Salle University Senior High School (DLSU-SHS), the virtual simulation module would become an easy-to-access learning course which will not only teach them how to fly a drone, but also the components, parts and concepts behind drone technology. The student-friendly and engaging environment provided by the designed module will be able to help the students and encourage them to learn and discover more about drone technology in such a way that they will understand fully the module's contents. The module would also provide them basic knowledge before accessing more advanced modules.

The paper will present a new virtual simulation module for drone training in Senior High School that would prove to be engaging and beneficial for the students. Moreover, this study aims to incorporate a virtual game and 3D-printing technology in the module through the printing of drone parts that will be made available on the website. The researchers plan to test the performance and efficiency of the module when introduced to a random selection of senior high school students. 


\section{Virtual Simulations for Drone Education of Senior High School Students}

\section{METHODOLOGY}

The methodology developed in the paper could be seen in Fig. 1. It all started with the development of the website and the incorporation all the information and activities for the virtual simulations.

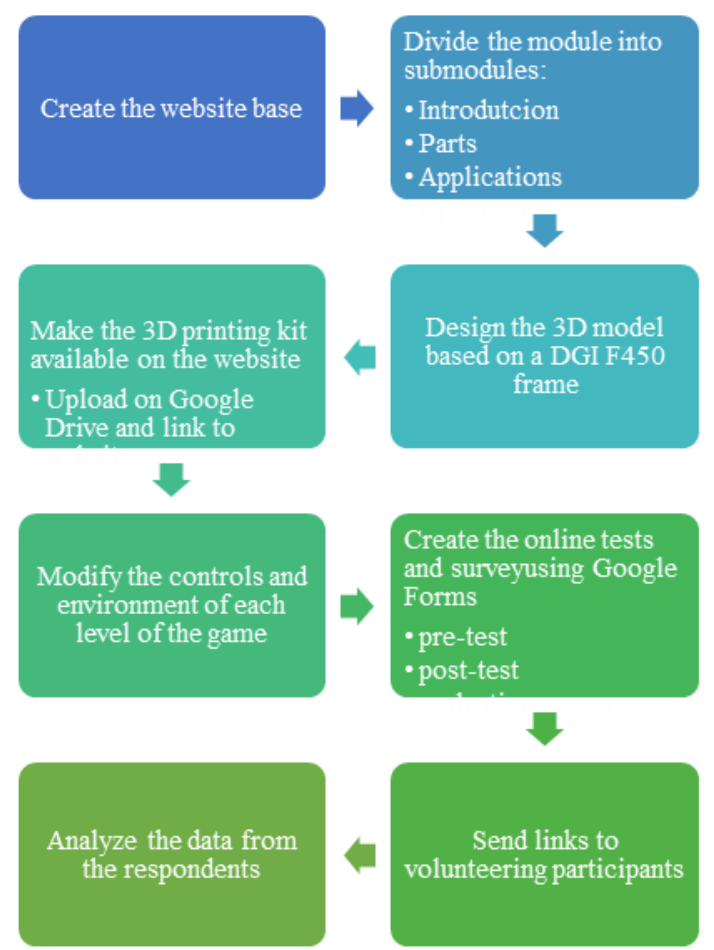

Fig. 1 Step-by-step procedure of the Methodology

\section{A. Website}

The researchers used a web design service to combine learning elements and create a website for the drone training module. This website is the most important part of the virtual simulation module since this will be the base for the other components, i.e. the simulation game and the 3D printing kit Wix was the preferred web design service so that the website is user-friendly and easy to manage. The elements within the website included video tutorials, pictures and slideshows, an interactive game, and 3D-printer designs of a demonstration drone provided and created by the researchers.

\section{Submodules}

The researchers first compiled the information they wanted to include in the module, created learning objectives for the entire course and divided that information into submodules.

\section{(a) Introduction}

This course discussed the definition of UAVs, specifically of quadcopters. It also discussed the history and evolution of UAVs, most common types, uses and applications. The learning elements included here were pictures and a YouTube video about the history of drones from Mashable, which linked to the website.

\section{(b) Parts and Functions}

This course tackled the basic parts and functions of a standard quadcopter drone. The learning elements included here were pictures of each part to help the users familiarize themselves with each part. This course also featured important notes on specific parts, such as the motors, propellers and configuration of the drone, which when altered can affect how the drone operates.

\section{(c) Operation Basics}

This course showed the basic concepts of flying a drone, which are the pitch, roll, yaw and throttle movement. To help the users visualize the movements, animated pictures or GIFs were used.

\section{Website Framework}

After compiling the information and organizing the courses, the researchers created a flowchart of the website's framework to have a better overview of where to insert links, where each page opens to and what content to put there. The website framework can be summarized in a chart such shown in Fig. 2.

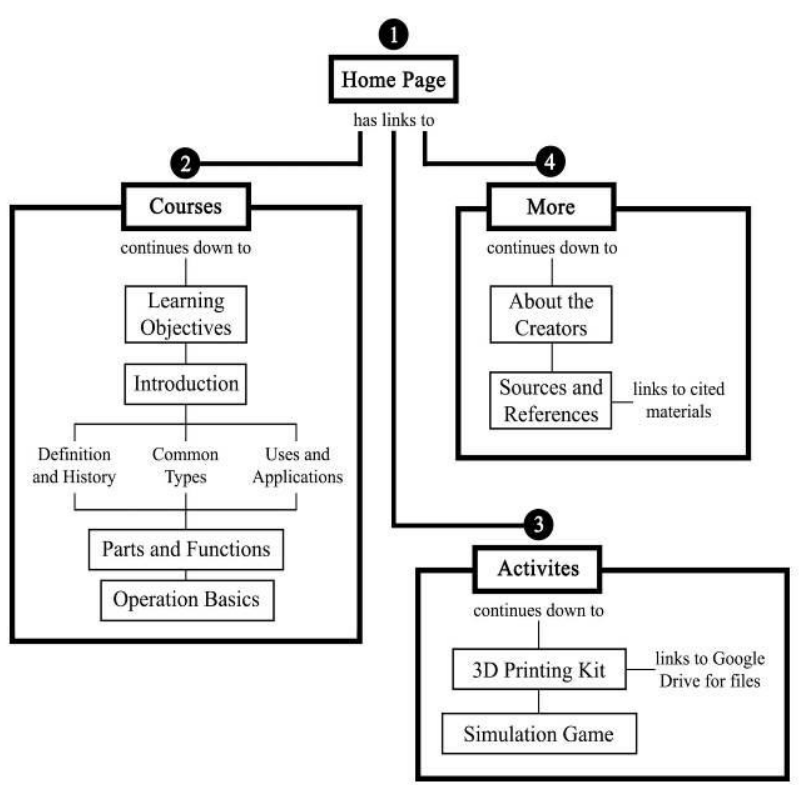

Fig. 2 Website Framework

(a) Home Page

This home page (Fig. 3) features a sneak peek of the contents of the module, which also contains links that lead the user to a specific page.

\section{DRONE TRAINING MODULE} FOR SENIOR HIGH SCHOOL

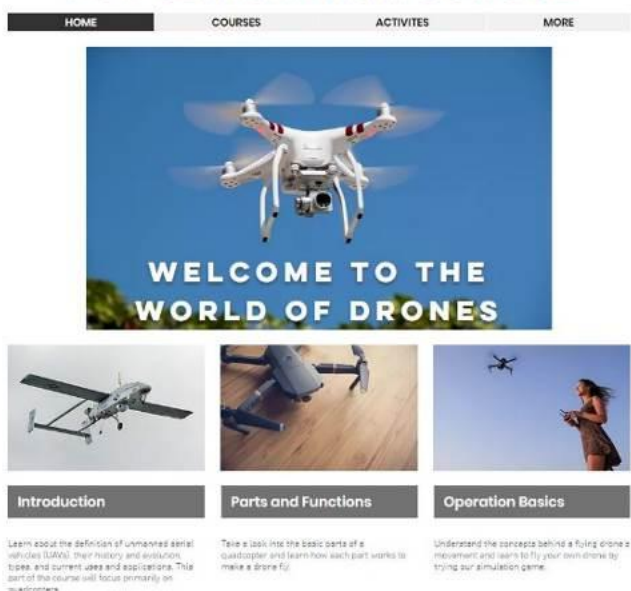

Fig. 3 Home Page [10]-[13]

Published By: 


\section{(b) Courses}

This page is a continuous page; that is, all the information can be viewed by scrolling down on the current page. This contain the learning objectives, introduction, and others. There is, however, a menu at the bottom right corner of the screen so that the users are able to skip quickly to the part they wish to read.

\section{(c) Activities}

This page (see Fig. 4) contains the 3D printing kit and the simulation game, which are one of the key components of the module. The 3D printing kit is accessible through a link that directs the user to a Google Drive, where the printing files are stored and ready for download. This section also features pictures of the 3D model's individual parts so that the users have an idea of what the model looks like per part and as assembled. The simulation game is embedded into the website for ease of access to the user, and above that is a short introduction to the game explaining its objectives and how to control the game drone.

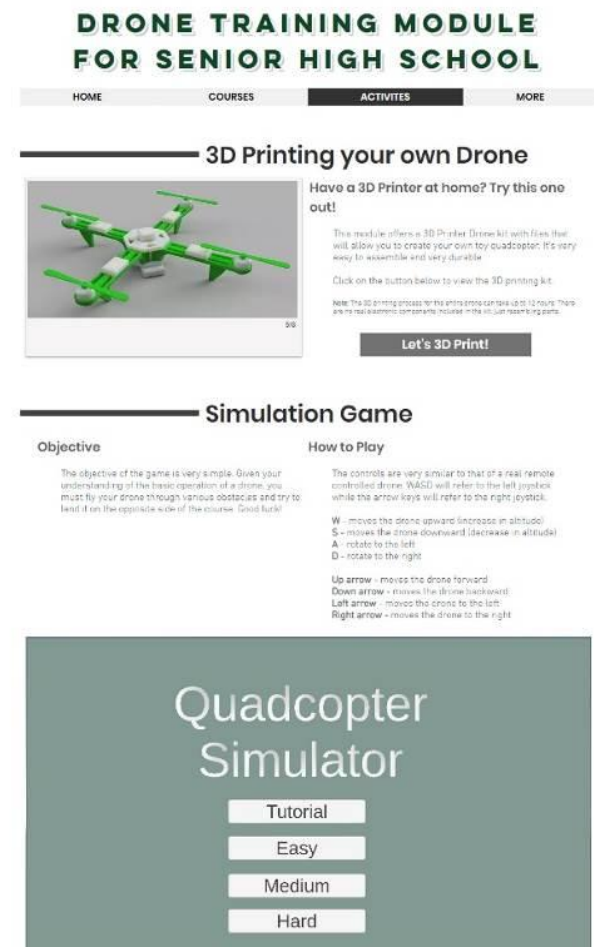

Fig. 4 Activities Page

\section{B.Drone Virtual Simulation Game}

The interactive game was created using the game development engine Unity. Since Unity was complex for the understanding and capabilities of the researchers to start from scratch, they bought a pre-made drone flying kit complete with a drone model, an editable environment and functional controls. The controls were modified and decentralized in such a way that it mimicked a real drone RC transmitter (i.e., the left joystick movements will correspond to WASD, while the right joystick movements will correspond to the arrow keys).

Fig. 5 to 8 shows the environment of the different modes of the game from tutorial level including the easy, medium and difficult level of playing the game. .

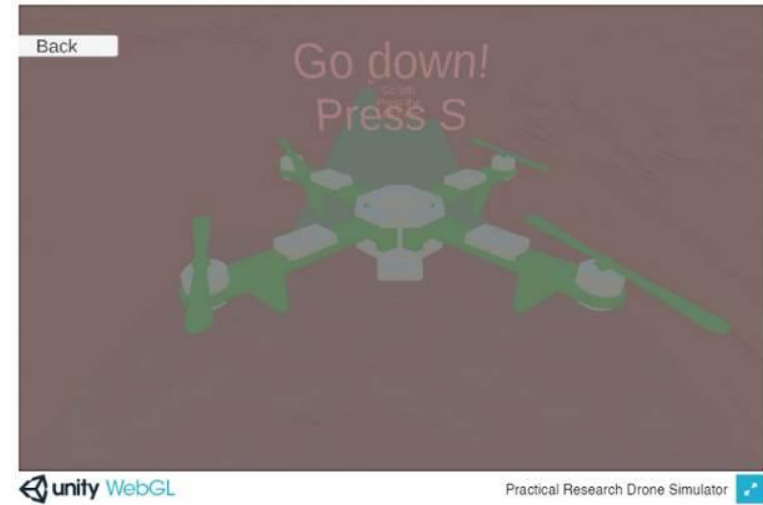

Fig. 5 Tutorial Level

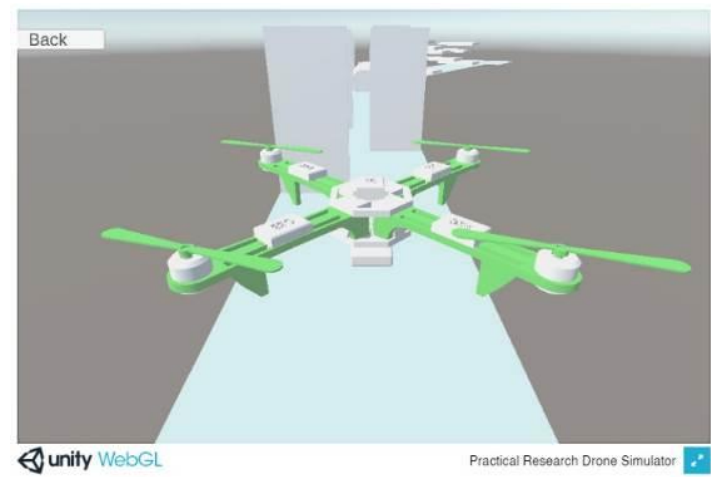

Fig. 6 Easy Level

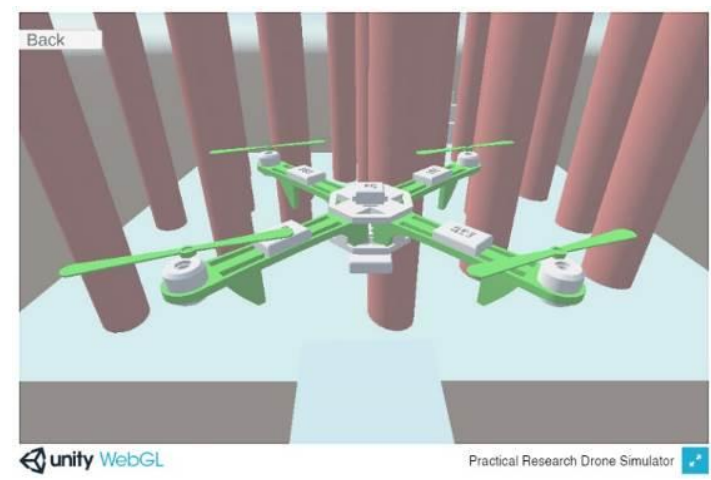

Fig. 7 Medium Level

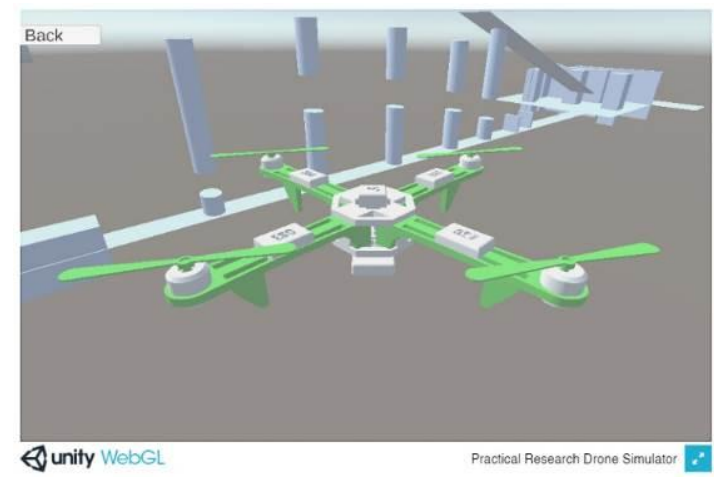

Fig. 8 Difficult Level

\section{C.3D Printing}

The 3D printed demonstration drone (Fig. 9) will have only the basic components, like the frame, the legs and the rotors, and not the electronic components to make it fly. Mock-ups, however, will be provided as placeholders for the

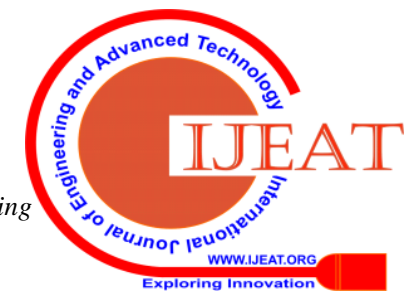


real electronic components so as to give the users a better overview of where the electronic components are attached to the drone. The software to be used for designing the demonstration drone is Autodesk Fusion 360 and will be printed after going through a model slicing program.

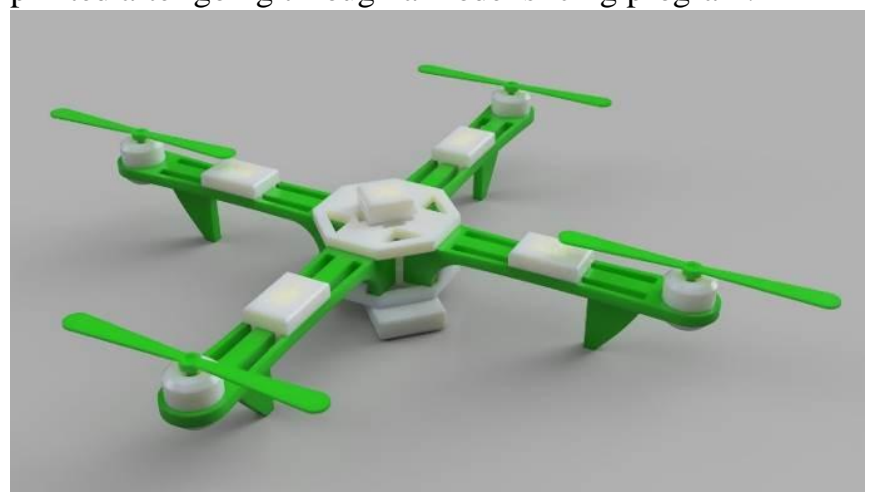

Fig. 9. 3D Model, assembled and rendered

\section{D.Survey}

To be able to test the drone module's effectiveness, the researchers conducted a series of tests among a conveniently selected sample after guiding the said sample through the created module. These tests included a survey, constructed specifically to aid in the research. A pre-test and post-test were also given to the sample to see how much they knew prior to their experience with the module and how much they learned from it after the experience.

These tests were able to provide the researchers data on what parts of the module that the respondents deemed effective and ineffective, satisfactory and in need of development.'

\section{Evaluation Survey}

This was divided into four sections: website evaluation, game evaluation, 3D model evaluation and feedback section. The first three sections were a series of Likert Scale items with a 5-point system while the feedback section contains a rating of the three components of the module (website, game, model), a rating of the easiest and most difficult parts of the module, and an open-ended question for the users' feedback and comments.

\section{Pre-test and Post-test}

These tests compose of 15 questions which are taken from the contents of the website. They are identical tests, with the only difference being that the pre-test is administered before studying the module and the post-test is administered after the user has completed the module.

\section{RESULTS}

Under this section, the data collected from the methodology of the research will be tallied and analyzed statistically. The following results are based on 22 responses, and are taken from the evaluation survey, which was divided into four main parts: website evaluation, game evaluation, 3D model evaluation and general evaluation.

The results of survey, with the exception of the general evaluation, were analyzed through paired t-testing, a statistical technique used to determine whether the mean difference between two sets of data is zero. This best analyzes the effectiveness of the module by means of using the scores collected from the pre-test and post-test, and positive versus negative responses for the website, game and 3D model evaluation

The survey parts for the website, game and 3D model all compose of a 5-point Likert Scale. To analyze the data, the researchers grouped the scale of 5-4 (which corresponds to 'strongly agree' and 'agree' respectively) as the positive reviews which indicate that that specific part of the module was effective and to their liking. The positive reviews group will be tested against the negative reviews group, which comprise of the 2-1 scale (which corresponds to 'disagree' and 'strongly disagree' respectively). The neutral group of scale 3 (which corresponds to 'somewhat) will not be included in the testing.

\section{A. Website}

The survey for the website comprised of 13 questions. Out of all the responses, $91 \%$ were positive, $1 \%$ were negative while $8 \%$ were neutral. After the paired t-test, the resulting p-value was $0.25 \times 10-14$, which is less than the significance level 0.05. Given this data, the action to take is to reject the null hypothesis, which states that there is no significant difference between the positive and negative responses. Therefore, at $95 \%$ confidence, with $\mathrm{p}=0.25 \times 10-14$, it can be concluded that there is a significant difference between the positive and negative responses to the survey. Please see Table I for the details of the t-test done.

Table I. t-Test: Paired Two Sample for Means (Website Analysis)

\begin{tabular}{lrr}
\hline & \multicolumn{1}{c}{5,4} & \multicolumn{1}{c}{2,1} \\
\hline Mean & 20.23076923 & 0.153846154 \\
Variance & 1.358974359 & 0.307692308 \\
Observations & 13 & 13 \\
Pearson Correlation & -0.317220634 & \\
Hypothesized Mean Difference & 0 & \\
df & 12 & \\
t Stat & 50.22947342 & \\
P $(T<=t)$ one-tail & $1.2717 \mathrm{E}-15$ & \\
t Critical one-tail & 1.782287556 & \\
P $(T<=t)$ two-tail & $2.5434 \mathrm{E}-15$ & \\
t Critical two-tail & 2.17881283 & \\
\hline
\end{tabular}

Based on the survey questions and the number of positive responses, the website is truly commended for its easily readable information and correct usage of grammar. Other areas with the most positive responses were the use of language and appropriation of technical terms with regard to the targeted audience, the website's functionality in terms of properly working links and syntax errors, and the content's informative quality. There were neutral responses on the appeal and aesthetic of the website itself, pertaining to its design. The only negative response came from the interactive extent of the website, which will be further clarified by the feedback in the General Evaluation part below. 


\section{B.Game}

The survey for the simulation game comprised of 10 questions. Out of all the responses, $66 \%$ were positive, $8 \%$ were negative while $26 \%$ were neutral. After the paired t-test, the resulting p-value was $0.29 \times 10-6$, which is less than the significance level 0.05 . Given this data, the action to take is to reject the null hypothesis, which states that there is no significant difference between the positive and negative responses. Therefore, at $95 \%$ confidence, it can be concluded that there is a significant difference between the positive and negative responses to the survey (see Table II).

Table II. t-Test: Paired Two Sample for Means (Game Evaluation)

\begin{tabular}{lrr}
\hline & 5,4 & 2,1 \\
\hline Mean & 14.5 & 1.7 \\
Variance & 4.944444444 & 1.122222222 \\
Observations & 10 & 10 \\
Pearson Correlation & -0.636785369 & \\
Hypothesized Mean Difference & 0 & \\
df & 9 & \\
t Stat & 13.44268881 & \\
$\mathrm{P}(\mathrm{T}<=\mathrm{t})$ one-tail & $1.45574 \mathrm{E}-07$ & \\
t Critical one-tail & 1.833112933 & \\
$\mathrm{P}(\mathrm{T}<=\mathrm{t})$ two-tail & $2.911475 \mathrm{E}-07$ & \\
$\mathrm{t}$ Critical two-tail & 2.262157163 & \\
\hline
\end{tabular}

Based on the survey questions and the number of positive responses, the most positive responses were from the game's ability to motivate the user to engage themselves in the experience, the learning obtained about the operation of a drone, and the overall experience of playing being worthwhile to the user. However, there were neutral responses on how easy it was to learn the objectives and controls of the game. The most negative responses came from the aesthetic of the game's design and the user being able to grasp the controls of the game.

\section{C.3D Printing}

The survey for the 3D printed model comprised of 11 questions. Out of all the responses, $88 \%$ were positive, $2 \%$ were negative while $10 \%$ were neutral. After the paired t-test, the resulting p-value was $0.71 \times 10-9$ (see Table III), which is less than the significance level 0.05 . Given this data, the action to take is to reject the null hypothesis, which states that there is no significant difference between the positive and negative responses. Therefore, at $95 \%$ confidence, it can be concluded that there is a significant difference between the positive and negative responses to the survey.

Based on the survey questions and the number of positive responses, the most positive responses were on the quality and consistency of the material used in 3D printing the drone model, and the distinguishability of each part of the model compared to the actual parts of a drone (which was discussed in the website). However, in terms of wanting to display their own 3D model at home, there were more neutral responses that negative responses.

\section{D.Surveys}

Pre-test and Post-test
The pre-test and post-test comprised of 15 items each. The two tests are identical, with the only difference being that the pre-test is administered first, and then the post-test after the participant has completed the module. Below is Table IV that summarizes the mean, median and range of scores for both the pre-test and the post-test.

The above states that the post-test garnered higher scores than the pre-test did. Statistically speaking, the given significance level is 0.05 , while the obtained p-value is $0.74 \mathrm{x}$ $10-4$. Since $\mathrm{p}$-value $<0.05$, the action to take is to reject the null hypothesis. Therefore, at $95 \%$ confidence level, it can be concluded that there is a significant difference between the pre-test and post-test scores. Based on the results, this means that the users learned more about drones through the module.

Table III. t-Test: Paired Two Sample for Means (3D-printed Drone Model Evaluation)

\begin{tabular}{lrr}
\hline & \multicolumn{1}{c}{5,4} & 2,1 \\
\hline Mean & 19.27272727 & 0.454545455 \\
Variance & 4.818181818 & 0.472727273 \\
Observations & 11 & 11 \\
Pearson Correlation & -0.819217652 & \\
Hypothesized Mean Difference & 0 & \\
df & 10 & \\
t Stat & 22.39965916 & \\
P $(T<=t)$ one-tail & $3.53484 \mathrm{E}-10$ & \\
t Critical one-tail & 1.812461123 & \\
P(T<=t) two-tail & $7.06969 \mathrm{E}-10$ & \\
$\mathrm{t}$ Critical two-tail & 2.228138852 & \\
\hline
\end{tabular}

Table IV. Pre-test and Post-test Scores

\begin{tabular}{rccc}
\hline & Mean & Median & Range \\
\hline Pre-test & 10 & 10 & $7-14$ \\
Post-test & 12 & 12 & $8-15$ \\
\hline
\end{tabular}

\section{General Evaluation}

In the first question, where the respondent is asked to rank the most interesting component of the module, $45 \%$ agreed that the simulation game piqued their interest the most while it was a tie between the website and the 3D model at $41 \%$.

The respondents were also asked to enumerate the easiest and most difficult parts of the module according to each's opinion. Majority answered that the easiest part of the module was the introduction to drone technology, while the most difficult part was a tie between understanding the concepts behind drone operation and controlling the drone in the simulation game.

Fig. 10 shows a graph of the easiest and most difficult parts of the virtual simulation module. This shows that easiest part is the introduction while the most difficult the understanding of the concepts of operation. 


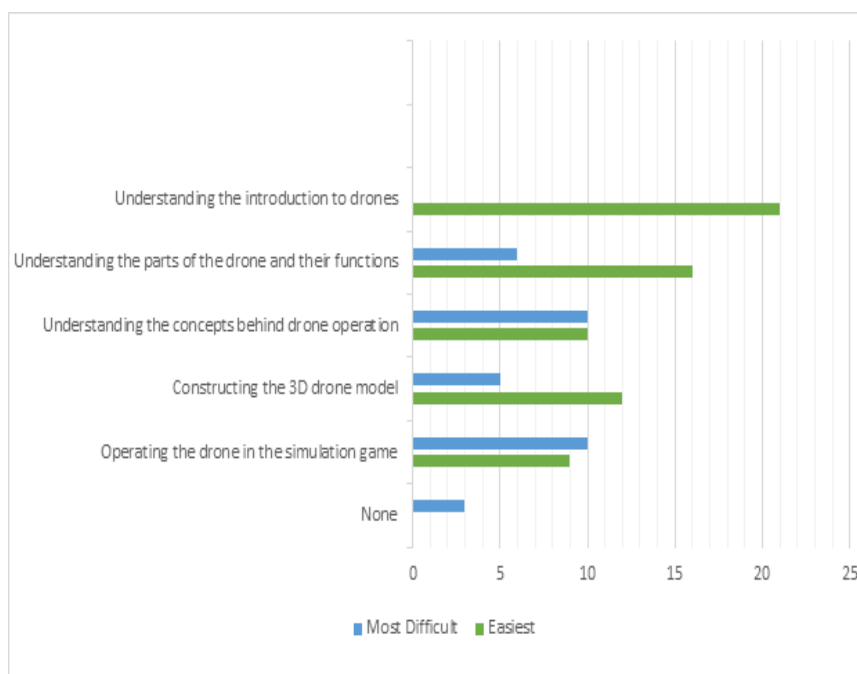

Fig. 10. Graph showing the Easiest (green) and Most Difficult (blue) parts of the module

The general evaluation section is also where the comments and feedback can be found. The most common feedback are as follows:

- Improve the simulation game (e.g. more in-depth objectives, smoother and more engaging graphics, better controls, navigation between levels and the main menu)

- Improve the 3D model (e.g. making it bigger and sturdier)

- Add more interactive elements to the website to make it more enticing (e.g. pictures, videos)

- Add more depth to the module (e.g. expounding on RC transmitters and receivers)

- Make the module mobile friendly

- Make the simulation game mobile so that it's more interesting to play

\section{CONCLUSIONS}

In conclusion, the use of a virtual simulation module for drone education is very effective. Not only does it provide conveniently accessible information at any given time, it also provides the students unique learning materials, such as the $3 \mathrm{D}$ printing kit and the simulation game. However, even though the module can easily be learned independently, the researchers still highly suggest that drone education be integrated into the K-12 curriculum through robotics classes, where a physical instructor is present to direct the class.. Based on the results of the study, students preferred a more technical approach to the topic of drone education, and what better way to help them learn than through an instructor who experts in the field of robotics, specifically in drone technology.

\section{ACKNOWLEDGMENT}

The authors would like to acknowledge the Department of Science and Technology- PCIEERD and the Mechanical Engineering Department of De La Salle University for supporting the research project.

\section{REFERENCES}

1. Michel, A. (2015). Drones in Popular Culture. Center for the Study of the Drone. $\quad$ Retrieved from http://dronecenter.bard.edu/drones-popular-culture/

2. Olivares-Mendez, M., et. al. (2015). Towards an Autonomous Vision-Based Unmanned Aerial System against Wildlife Poachers. Sensors (Basel, Switzerland), 15(12), 31362-31391. Retrieved from http://doi.org/10.3390/s151229861

3. Civil Aviation Authority of the Philippines. (2012). MC 21-14 New Provisions to the Philippine Civil Aviation Regulations (PCAR) Part 1111.11 Unmanned Aircraft Vehicle (UAV) [PDF Document]. Retrieved from http://www.caap.gov.ph/?s=uav

4. Official Gazette. (n.d.). What is K to 12 Program? Official Gazette. Retrieved from http://www.officialgazette.gov.ph/k-12/

5. U.S. Department of Education. (2010.) Evaluation of Evidence-Based Practices in Online Learning [PDF Document]. Retrieved from https://www2.ed.gov/rschstat/eval/tech/evidence-based-practices/finalre port.pdf

6. Ord, J. (2012). John Dewey and Experiential Learning: Developing the theory of youth work. Journal of Youth \& Policy, 108. Retrieved from https://www.researchgate.net/profile/Jon_Ord/publication/270338098_ John_Dewey_and_Experiential_Learning_Developing_the_theory_of_ youth_work/links/54a7c6010cf267bdb90a3779/John-Dewey-and-Exp eriential-Learning-Developing-the-theory-of-youth-work.pdf

7. Global Tree. (2017, November 6). Study Abroad Program for Better Future Career [blog post]. Retrived from https://globaltree.in/study-abroad-program-in-best-universities/

8. Gue, I, Chua, A (2018) Development of a Fuzzy GS-PID Controlled Quadrotor for Payload Drop Missions. Journal of Telecommunication, Electronic and Computer Engineering. Volume 10. Issue 1-5.

9. Cuevas, J, et al. (2017) Identification of River Hydromorphological Features using Viola-Jones Algorithm IEEE Region 10 Annual International Conference Pages 2300-2306

10. Picture of Fig. 3 :Plane http://www.navy.mil/view image.asp?id=25689

11. Picture of Fig. 3 :Playing https://www.pexels.com/photo/woman-playing-drone-1170344/

12. Picture of Fig. 3: Parts

https://www.pexels.com/photo/quadcopter-on-wooden-surface-1601217/ 13. Picture of Fig. 3: Drone

https://www.pexels.com/photo/drone-flying-against-blue-sky-336232/

\section{Authors Profile}

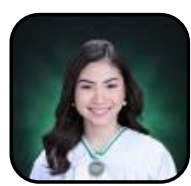

Julia Dominic P. Espinola is a graduate of Senior High School at De La Salle University, Manila, Philippines. As a student, she is a consistent honor student from elementary to high school and is planning to take up a science or engineering course in the future.. She has joined several competitions and have won several events that shows her versatility. She is also fond of joining different organizations to develop her skills in different areas of her life. In her research, she focuses on research studies related to robotics, mechatronics and drone systems.

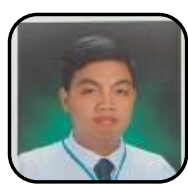

Jose Emmanuel D. Ignacio is a graduate of Senior High School at De La Salle University, Manila, Philippines. As a student, he is a consistent honor student from elementary to high school and is planning to take up a science or engineering course in the future.. He has joined several competitions and have won several events that shows his versatility. He is also fond of joining different organizations to develop his skills in different areas of his life. In his research, he focuses on research studies related to robotics, mechatronics and drone systems.

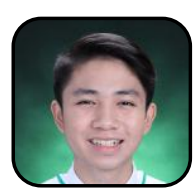

John Peter L. Lacaden is a graduate of Senior High School at De La Salle University, Manila, Philippines. As a student, he is a consistent honor student from elementary to high school and is planning to take up a science or engineering course in the future.. He has joined several competitions and have won several events that shows his versatility. He is also fond of joining different organizations to develop his skills in different areas of his life. In his research, he focuses on research studies related to robotics, mechatronics and drone systems. 


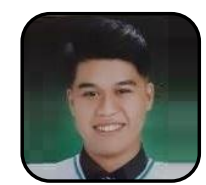

Charles Brian D. Toribio is a graduate of Senior High School at De La Salle University, Manila, Philippines. As a student, he is a consistent honor student from elementary to high school and is planning to take up a science or engineering course in the future.. He has joined several competitions and have won several events that shows his versatility. He is also fond of joining different organizations to develop his skills in different areas of his life. In his research, he focuses on research studies related to robotics, mechatronics and drone systems.

Alvin Y. Chua is a Full Professor and Current Chairman of the Mechanical Engineering Department of De La Salle University, Philippines. He earned his BSME, MSME, and Ph.D. in ME at De La Salle University-Manila. He conducted his dissertation research at the University of New South Wales, Australia. He received a special citation for the 2003 NAST-DuPont Talent Search for Young Scientists (Mechanical Engineering). He has published several papers in international conferences like Conference on Decision and Control (CDC), and Advance Intelligent Mechatronics (AIM). His research interests are on mechatronics, robotics, UAV, optimal estimation, and control 